\title{
The First Step for Neuroimaging Data Analysis: DICOM to NIfTI conversion
}

Xiangrui $\mathrm{Li}^{1}$, Paul S Morgan ${ }^{2}$, John Ashburner ${ }^{3}$, Jolinda Smith ${ }^{4}$, Christopher Rorden ${ }^{5}$

1.) Center for Cognitive and Behavioral Brain Imaging, The Ohio State University, Columbus $\mathrm{OH} 43210$, USA

2.) Medical Physics \& Clinical Engineering, Nottingham University Hospitals, Nottingham, UK

3.) Wellcome Trust Centre for Neuroimaging, University College London, London, United Kingdom

4.) Lewis Center for Neuroimaging, University of Oregon, Eugene OR 97403, USA

5.) McCausland Center for Brain Imaging, University of South Carolina, Columbia SC 29208, USA

Correspondence: Christopher Rorden, Department of Psychology, University of South Carolina, 29208, USA. Phone: +1 803404 2573. Email: rorden@sc.edu

\section{Highlights}

- DICOM to NIfTI conversion tools for different manufacturers and image modalities are introduced.

- The overview of the conversion basics useful for users and developers is covered.

- The methods to detect and correct problems of the conversion are presented, including those for slice order, phase encoding direction, diffusion gradient direction etc. 


\section{Abstract}

Background

Clinical imaging data are typically stored and transferred in the DICOM format, whereas the NIfTI format has been widely adopted by scientists in the neuroimaging community. Therefore, a vital initial step in processing the data is to convert images from the complicated DICOM format to the much simpler NIfTI format. While there are a number of tools that usually handle DICOM to NIfTI conversion seamlessly, some variations can disrupt this process.

New Method

We provide some insight into the challenges faced with image conversion. First, different manufacturers implement the DICOM format differently which complicates the conversion. Second, different modalities and sub-modalities may need special treatment during conversion. Lastly, the image transferring and archiving can also impact the DICOM conversion.

Results

We present results in several error-prone domains, including the slice order for functional imaging, phase encoding direction for distortion correction, effect of diffusion gradient direction, and effect of gantry correction for some imaging modality.

Comparison with Existing Methods

Conversion tools are often designed for a specific manufacturer or modality. The tools and insight we present here are aimed at different manufacturers or modalities.

Conclusions

The imaging conversion is complicated by the variation of images. An understanding of the conversion basics can be helpful for identifying the source of the error. Here we provide users with simple methods for detecting and correcting problems. This also serves as an overview for developers who wish to either develop their own tools or adapt the open source tools created by the authors.

Keywords: DICOM; NIfTI; Neuroimaging 


\section{Introduction}

Many of the popular tools used for scientific image processing, analysis and visualization require images to be stored in the NIfTI file format, whereas scanners used to acquire these images usually export data in the DICOM format. These two formats are each suited for their specific niche: DICOM is comprehensive and verbose, while NIfTI is simple and easy to support. Therefore, a common initial step in any neuroimaging analysis is to convert the images from DICOM to NIfTI format. Usually, a user can choose from one of many tools that seamlessly transforms their data. However, this step belies numerous challenges involved with this process. Specifically, the DICOM standard is particularly complicated, and different scanner manufacturers extend the DICOM standard in a variety of ways, often resulting in duplication of information and incompatibilities between software only designed to work with one particular subset of DICOM. Therefore, while one conversion tool may work for many images, it may fail for others. Our objective is to describe some of the general assumptions of these conversion tools and the situations where they may fail. Our primary aim is to inform users about how to identify these errors, and to provide suggestions for coping with these situations. We also provide an overview for developers who wish to either develop their own tools or adapt the open source tools created by the authors.

\section{DICOM format}

Modern medical imaging devices typically store data in the DICOM image format. The Digital Imaging and Communications in Medicine (DICOM) standard evolved from the American College of Radiology (ACR) and National Electrical Manufacturers Association (NEMA) standards, which originated in the 1980's (http://en.wikipedia.org/wiki/DICOM). The DICOM standard is complex, comprehensive (describing data transfer as well as compression), and evolving. For example, the 2011 edition of this standard now spans 4902 printed pages (http://dicom.nema.org/), which does not include specifics on details such as image compression. Annex A of Part 6 describes 37 different forms of transfer syntaxes (schemes for encoding image data). Further, section C.7.3.1.1.1 refers to 123 distinct modalities (some retired), with transfers of DICOM data often including DICOM files that include modalities such as scanned patient notes, audio files and other forms of embedded data. While our focus is on radiological modalities, when converting DICOM datasets to NIfTI the software will need to parse the neuroimaging DICOM files from any comingled DICOM files that store information from different modalities.

DICOM describes a tag based format, where each object in the file is encapsulated in a tag that describes the purpose and size of that chunk of data. Typically, the earlier objects store information about the participant, the device, the imaging sequence, and the image specifics (e.g. dimensions of the image), while the final object encodes the image data itself. Each object contains, in order, a Tag, an optional Value Representation (VR), a Length and the Value of the object itself (Table 1).

A Tag is a two-number code (e.g. "0028, 0010") defining the meaning of the element. What the element exactly means relies on a so-called DICOM dictionary. The DICOM standard defines a dictionary for a large number of variables, referred to as the public tags. In addition, the standard also allows manufacturers to define their own tags, so-called private tags. The first element of the two-number code of public tags is always even, whereas it is odd for the vendor-specific private tags. This ensues the 
private tags won't conflict with public tags, but different vendors may use the same block of private tags for different purpose.

The optional second component in an object, VR, is a two-character code defining the data type of the value in the object. For example, "US" refers to unsigned short integer(s), "DS" refers to decimal string, etc. Whether or not the VR field is present in the file depends on the transfer syntax, which may be either "implicit" or "explicit". The VR is encoded in the file for the explicit transfer syntax, whereas for the implicit representation, the VR needs to be determined from the tag using a dictionary. For implicitly represented private tags, the VR may not be available (or at least very difficult to track down), which makes the data very difficult to interpret. Although most modern DICOM files adopt explicit VR, the default DICOM standard involves implicit VR. A further complication relates to whether explicit transfer syntax is big- or little-endian.

The next component (Length) defines number of bytes of the stored value, while the final component encodes the Value itself. The Length provides the information needed to determine where the next object in the file begins.

There can be many objects in a DICOM file (typically about a hundred, but there can be three orders of magnitude more for multi-frame DICOM). Table 1 shows several important objects that are usually used for DICOM to NIfTI conversion.

Table 1: Some important DICOM objects for interpreting image data and converting to NIfTI. The Length and Value fields show some fairly typical values.

\begin{tabular}{|l|l|l|l|l|}
\hline Tag & Meaning & VR & Length & Value \\
\hline$(0002,0010)$ & Transfer Syntax UID & UI & 20 & '1.2.840.10008.1.2.1' \\
\hline$(0018,0050)$ & Slice Thickness & DS & 2 & 3 \\
\hline$(0018,0088)$ & Spacing Between Slices & DS & 16 & 3.0 \\
\hline$(0018,5100)$ & Patient Position & CS & 4 & 'HFS' \\
\hline$(0020,0011)$ & Series Number & IS & 2 & 2 \\
\hline$(0020,0013)$ & Instance Number & IS & 2 & 1 \\
\hline$(0020,0032)$ & Image Position Patient & DS & 50 & $-100-120-40.2$ \\
\hline$(0020,0037)$ & Image Orientation Patient & DS & 102 & $100 \quad 0 \quad 10$ \\
\hline$(0028,0010)$ & Rows & US & 2 & 64 \\
\hline$(0028,0011)$ & Columns & US & 2 & 64 \\
\hline$(0028,0030)$ & Pixel Spacing & DS & 4 & $3 \quad 3$ \\
\hline$(7 f e 0,0010)$ & Pixel Data & OW & 10952 & \\
\hline
\end{tabular}

The Transfer Syntax UID encodes important information about how to decipher the rest of the DICOM file. In particular, it defines the transfer syntax, as well as information about how image data may be compressed. The Rows, Columns, and maybe other elements define the dimension of the Pixel Data. Usually, each DICOM file encodes a single 2D slice, although there are exceptions to this general rule. The Image Position and Orientation in patient coordinate define the slice location and orientation in the scanner space. The Pixel Spacing and Spacing Between Slices (or Slice Thickness) defines the voxel 
size in three dimension. The Series and Instance Numbers, and maybe others, help sort the DICOM files. Sometimes, some of these objects are missing from the DICOM files, so heuristic approaches are needed to try to determine which slices should go together in the same 3D volumes.

\section{NIfTI format}

As noted, the DICOM standard has proved hugely successful, providing a unified framework for transferring, storing and printing medical data. While DICOM is very flexible and comprehensive, it does require considerable effort and expense to implement transparently. Research environments usually lack the resources for such undertakings, which also aligns poorly with the continual advances in analysis methods and data. Instead, researchers usually use simpler image formats that allow more rapid progress to be made. These simpler formats retain only a limited, relevant set of the images' metadata.

In contrast to DICOM, NIfTI is a very simple, minimalistic format. This format has been widely adopted in neuroimaging research, allowing scientists to mix and match image processing and analysis tools developed by different teams. The NIfTI format was originally designed to be a backwardscompatible extension of the proprietary ANALYZE-7.5 file format (http://nifti.nimh.nih.gov/nifti-1). C libraries have been developed by the community to read and write NIfTI files, which means that developers do not need to re-implement support to this format (which is not only time consuming, but also allows the opportunity to introduce bugs).

The format specifies 348-bytes of header data and uncompressed image data. The header and image data can be saved as separate files (using the file extensions '.hdr' and '.img'), or as a single file (using the '.nii' extension, with the first 348 bytes devoted to the header and the image data typically beginning at byte 352). Images can have up to 7 dimensions (three spatial dimensions, time, and then other dimensions such as diffusion gradient direction). Note that the space dimensions do not have to be in the order of left-right, posterior-anterior and inferior-superior, although this is normally true for axially oriented acquisitions. One improvement of NIfTI over its predecessor, Analyze format, is that it allows spatial orientation information to be stored more fully. Therefore, NIfTI-compliant software should reduce the chance of making left-right errors. Specifically, NIfTI images can include two independent spatial transforms for mapping the image data into different frames of reference. One of these, the "sform", allows a full 12-parameter affine transform to be encoded, whereas the other one, the "qform", allows only a 9-parameter mapping. The latter transform is limited to encoding translations, rotations (via a quaternion representation) and isotropic zooms. While it is suitable for mapping voxels in most MRI images to some Euclidean coordinate system, the 9-parameter transform is unable to encode the shears that are often needed to account for the gantry tilt of CT scanners.

The inclusion of two different spatial transforms in the NIfTI header can also cause some confusion. When both sform and qform representation are included, some tools, such as MRIcron and SPM, give precedence to the sform, while others (e.g. those relying on the Insight Segmentation and Registration Toolkit, http://www.itk.org) default to the qform. Therefore, the same image can appear differently in different viewers and the starting estimates for image registration may differ among tools. 
While the simplicity of NIfTI is a major advantage, it necessarily constrains what can be stored in the header, which, as we will see, can lead to confusion with some sequences (specifically, diffusion imaging and multi-band sequences). NIfTI format does allow extensions to encode such information, but consensus among the community would be needed for these extensions to be used in a consistent way. For this reason, many packages (e.g., SPM) simply ignore any extensions.

Table 2 shows some example parameters in the NIfTI header that users may need to be aware of.

The "dim" field encodes the dimension of image data. The first number indicates how many dimensions. For the example in Table 2, the image has four dimensions. The next four numbers indicate the size of each dimension. Typically, the first three dimensions are for space, and fourth one is for time (although this convention is often abused).

Table 2: Several important NIfTI header items. Each item has fixed length, and fixed location inside the header.

\begin{tabular}{|c|c|c|}
\hline Name & Meaning & Value example \\
\hline $\operatorname{dim}$ & Image dimension & 4646438200111 \\
\hline pixdim & Voxel size and time interval & $\begin{array}{lllll}13 & 3 & 3 & 2 & 000\end{array}$ \\
\hline slice_code & Slice order code & 0 to 6 \\
\hline descrip & Human readable text & 'time $=091230 ;$ phase $=-\mathrm{y} ;{ }^{\prime}$ \\
\hline $\begin{array}{l}\text { quatern_b } \\
\text { quatern_c } \\
\text { quatern_d }\end{array}$ & $\begin{array}{l}\text { Quaternion b, c, d } \\
\text { parameters }\end{array}$ & $\begin{array}{l}0 \\
0 \\
0\end{array}$ \\
\hline $\begin{array}{l}\text { qoffset_x } \\
\text { qoffset_y } \\
\text { qoffset_z }\end{array}$ & $\begin{array}{l}\text { Quaternion transform } \\
\text { offset }\end{array}$ & $\begin{array}{l}-100 \\
-120 \\
-40.2 \\
\end{array}$ \\
\hline $\begin{array}{l}\text { srow_x } \\
\text { srow_y } \\
\text { srow_z }\end{array}$ & $\begin{array}{l}\text { Three rows of affine } \\
\text { transform }\end{array}$ & $\begin{array}{llll}3 & 0 & 0 & -100 \\
0 & 3 & 0 & -120 \\
0 & 0 & 3 & -40.2\end{array}$ \\
\hline
\end{tabular}

The "pixdim" encodes the voxel size and time interval corresponding to the spatial and temporal dimensions. The first value has a special purpose, which will be mentioned later. The units of voxel size and time are coded in parameter "xyzt_units", but most converters default to millimeters and seconds.

The "slice_code" is useful for slice timing correction. It specifies the slice order. Codes 1 through 4 refer to ascending, descending, interleaved ascending, and interleaved descending respectively. Codes 5 and 6 are for interleaved ascending and descending, but starting with the 2 nd and 2 nd to the last slice. DICOM files do not always encode the information needed for the slice_code, so converters may not fill in this field.

The "descrip" field can hold up to 80 characters, and is often used to store some textual description of the image that does not fit in the NIfTI header. In the example in Table 2, it is likely the acquisition start time and phase encoding direction. The former may be used to align with some physiological recording, and the latter may be useful for image distortion correction. 
The qform and sform information are the two methods for encoding affine mappings mentioned earlier. The example in Table 2 shows an axial acquisition without any tilt, which produced zeros for all three quaternion parameters, and pixdim values (three 3s) for diagonal of affine transform. If there is small tilt from the major axes, those $3 \mathrm{~s}$ may be a little smaller than 3 , and those zeros may become numbers with small absolute value. The three qoffset parameters are the offset at three space dimensions, and they are the same as the last column of sform transformation matrix if sform and qform encode the same coordinate system.

One major variant of these parameters is worthy of mention. For the axial acquisition example in Table 2, those transform parameters may be very different even for the same dataset. Often, the three quaternions are $[0-10]$ or close to that, and the srow_x has a -3 rather than 3 for the first number. This is the indication that the image data is stored in so-called left-hand storage. This means that the same dataset can be organized in different way and with different transform parameters. If they are overlaid in an image viewer they will match perfectly. However when one applies analysis to the NIfTI image data, be aware of this variant, and deal with the image flip accordingly if needed.

For slice orientation other than axial, the image could be re-organized as if it were axial slice. This won't cause any problem in terms of the NIfTI format, and may avoid confusion in some analysis and visualization tools. However, there are other considerations against doing so. A major concern is due to the fact that most analysis tools treats the third dimension as slice dimension, and may not be able to perform slice timing correction correctly otherwise. If the first number in column 3 of the affine transform has large absolute value in the example in Table 2, that means the third dimension of the image data is along left-right axis, and accordingly the fourth numbers in dim and pixdim are for this axis. If there was no image data re-organization, this indicates the sagittal slice acquisition.

\section{Methods}

\section{DICOM to NIfTI conversion}

To convert DICOM into NIfTI, the first step is to sort files into different series. A DICOM series includes a set of DICOM images (Instances) that were generated together by the same equipment at the same operation. The most reliable way to sort series is by DICOM object Series Instance UID, although it can also be done by combining objects Patient Name, Study ID, and Series Number. Within each series, we need to sort the images into different volumes, if applicable. This is typically identified by Instance Number. For some types of files, one or more of the following objects may be needed to reliably sort images within a series: Acquisition Number, Echo Number, Image Position Patient, Image Type and others. Then the images within a series can be stacked into an image with up to 7 dimensions.

The spatial transform parameters are of great importance in the NIfTI header. Mathematically, a transformation matrix is used to project voxel indices to a location in a coordinate system, Patient Coordinate System (PCS, Figure 1) for DICOM. The following DICOM objects are normally needed to construct the transformation matrix. 
Figure 1: DICOM Patient Coordinate System (PCS) and NIfTI coordinate system when Patient Position is Head First Supine (HFS). The arrows of axes indicate the positive directions, LPS for DICOM and RAS for NIfTI. For DICOM images in the MR modality, the origin (coordinate $0,0,0$ ) refers to the magnet isocenter. For DICOM CT it refers to the table center. After warping to standard space, the NIfTI format specifies that the origin should be set to the location of the anterior commissure. Note that, despite the axis direction difference, both DICOM and NIfTI coordinates systems are so-called right-handed systems, which is different from Analyze coordinate system.

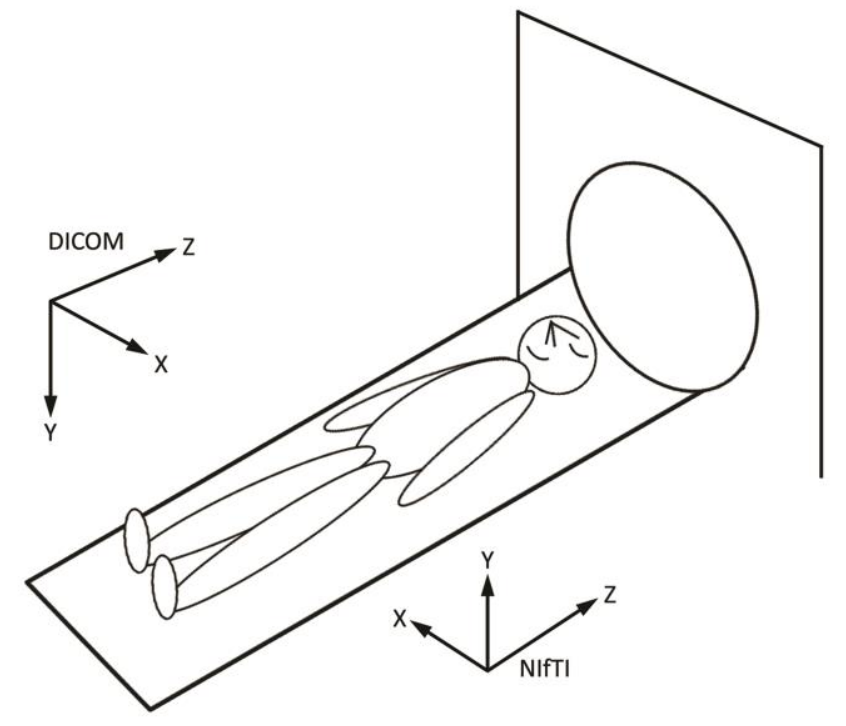

The Image Orientation Patient (see Table 1) contains two triplets, $\left[r_{x} r_{y} r_{z} c_{x} c_{y} c_{z}\right]$, which encode direction cosines of the row and column of an image slice. The Image Position Patient of the first slice in a volume, $\left[x_{1} y_{1} z_{1}\right]$, is the $x, y, z$ coordinates of the upper-left corner voxel of the slice. These two parameters define the location of the slice in PCS. To determine the location of a volume, the Image Position Patient of another slice is normally needed. In practice, we tend to use the position of the last slice in a volume, $\left[x_{n} y_{n} z_{n}\right]$. The voxel size within the slice plane, $\left[v_{r} v_{c}\right]$, is stored in object Pixel Spacing. The transformation matrix in DICOM coordinates can be constructed by Equation 1 (where $n$ is the number of slices in a volume):

$$
R_{\text {DICOM }}=\left[\begin{array}{rrrr}
r_{x} v_{r} & c_{x} v_{c} & \left(x_{n}-x_{1}\right) /(n-1) & x_{1} \\
r_{y} v_{r} & c_{y} v_{c} & \left(y_{n}-y_{1}\right) /(n-1) & y_{1} \\
r_{z} v_{r} & c_{z} v_{c} & \left(z_{n}-z_{1}\right) /(n-1) & z_{1} \\
0 & 0 & 0 & 1
\end{array}\right]
$$

This method also has the advantage to account for possible shears in some CT data along slice direction (see Section CT Modality).

If the location information of the last slice, $\left[x_{n} y_{n} z_{n}\right]$, is not conveniently available for a dataset, we need to use another method for the third column of the matrix. This is the case for Siemens mosaic images (see Section Siemens DICOM). 
To get the transformation matrix in NIfTI coordinates $\left(R_{\text {NIfTI }}\right.$ in Equation 2$)$, we need to change the signs of the first two rows in $R_{D I C O M}$, to reflect the definition difference of NIfTI coordinate system, which is RAS (right, anterior and superior) positive system (Figure 1). The first three rows of $R_{\text {NIfTI }}$ are srow_X, srow_y and srow_z (Table 2) for the NIfTI header. The quaternion parameters can be computed from $R_{\text {NIfTI. }}$ Note that the quaternion b, c and d cannot determine the sign of slice direction, so the sign is stored in the first number of pixdim (Table 2).

$$
R_{N I f T I}=\left[\begin{array}{rrrr}
-r_{x} v_{r} & -c_{x} v_{c} & -\left(x_{n}-x_{1}\right) /(n-1) & -x_{1} \\
-r_{y} v_{r} & -c_{y} v_{c} & -\left(y_{n}-y_{1}\right) /(n-1) & -y_{1} \\
r_{z} v_{r} & c_{z} v_{c} & \left(z_{n}-z_{1}\right) /(n-1) & z_{1} \\
0 & 0 & 0 & 1
\end{array}\right]
$$

The third column of Equations 1 and 2 also contains the slice thickness information. The slice thickness is normally defined by DICOM objects Spacing Between Slices or Slice Thickness (Table 1). However, we tend to rely on the Image Position Patient of two slices to robustly identify the distance between voxel centers. This arises for two reasons. First, by DICOM standard, Spacing Between Slices is only required for MRI, and not present for other neuroimaging modalities. Second, many systems incorrectly report the Spacing Between Slices to refer to the slice gap, rather than the combination of slice thickness and gap.

The transform in Equation 2 is sufficient by NIfTI standard. However, we may re-arrange the axes and/or flip image along one or more axes to better meet the requirement or resolve restriction of different analysis and visualization tools. The transforms will need to be updated accordingly.

In general, DICOM to NIfTI conversion tools will work well when dealing with 'normal' neuroimaging data. By normal we mean similar to the images observed by the teams who developed the image conversion tools. For example, most of these tools (dcm2nii, dicm2nii, MRIconvert, SPM) come from centers with only Siemens MRI systems. Therefore, a 'normal' conversion may be converting thousands of DICOM files to correctly parse and order the T1-weighted anatomical scan as a single 3D NIfTI file and a run of $\mathrm{FMRI}$ images as a second 4D NIfTI image. Unusual options might include different modalities (e.g. field-maps, diffusion, CT, etc.), storage to a DICOM server that compresses the image data, using a different vendor (e.g. GE or Philips). Below we describe how differences in vendor, modality, transfer and encoding influence conversion. Different tools (Table 3 ) are specifically designed to cope with some of these situations. 
Table 3: A comparison of some of the popular tools for converting images from DICOM to NIfTI (first row) and their capabilities. Features that are common to all tools are not listed (for example, each is available for OSX, Linux and Windows operating systems). Some tools are Matlab-based scripts while others are standalone executable. Some tools are able to decode compressed DICOM images (Compressed). Some tools support proprietary formats that pre-date DICOM (Legacy). Some tools support the Philips research format (PAR). Some tools support the unequal spacing and gantry tilt seen in computerized tomography (CT).

\begin{tabular}{|l|l|l|l|l|l|}
\hline Tool & Matlab & Compressed & Legacy & PAR & CT \\
\hline SPM & Yes & Limited & No & No & No \\
\hline MRIconvert & No & No & No & No & No \\
\hline dicm2nii & Yes & Limited & No & Yes & No \\
\hline dcm2nii & No & Limited & Yes & Yes & No \\
\hline dcm2niix & No & Yes & No & Yes & Yes \\
\hline
\end{tabular}

\section{Siemens DICOM}

Siemens DICOM for the MR modality is the most familiar to the developers of DICOM conversion tools, and Siemens sales brochures suggests (based on a Google scholar search) that it represents $70 \%$ of current 3T research (http://usa.healthcare.siemens.com/magnetic-resonance-imaging/mritechnologies/mri-services/trendsetting-applications). Two unique features of this format are the fact that some $3 \mathrm{D}$ volumes are stored as $2 \mathrm{D}$ mosaics, and the fact that some sequence details are not stored using public DICOM tags, but rather are saved in a private tag referred to as the CSA header (DICOM tag 0029,1010).

First, Siemens echo-planar imaging sequences (e.g. fMRI, DTI) typically store an entire volume as a single DICOM file storing a 2D mosaic, rather than a series of files each with a single 2D slice. In this configuration the data from a single slice are not stored in contiguous memory addresses. For example, consider the $6 \times 6$ mosaic shown in Figure 2. Here, the data for the first row is saved sequentially for the first six slices, then the data for the second row, and so on. The mosaic format reduces the number of files relative to $2 \mathrm{D}$ storage (one file per volume, rather than one file per slice), but these uncompressed images are wasteful of disk space (for example, volumes with 26 to 36 slices will be saved as a $6 \times 6$ mosaic and use the same disk space as an volume with 36 slices). The Siemens mosaic format was originally implemented to overcome disk writing overheads from storing the large number of files generated by $\mathrm{fMRI}$ acquisitions. Subsequently, an update to the DICOM standard implemented an extension to allow multiple image frames in a single DICOM file, which also addresses the waste of space in only partially filled Siemens mosaics. However, Siemens continue to use their mosaic format, possibly highlighting the inertia of updating their DICOM software in the light of the massive extent of the modern DICOM standard. Regardless, since NIfTI requires contiguous storage, the conversion software must correctly identify the true number of slices and reorder the image data. 
Figure 2: This image demonstrates an EPI acquisition saved as a $6 \times 6$ mosaic as generated by Siemens MRI scanners. The inferior slice in the top-left descending to the most superior slice toward the bottomright. Notice that this volume has 35 slices saved as a $6 \times 6$ matrix (the extreme bottom-right slot is blank). This image also shows the slice-timing order, where, on this scan, the participant's head was orthogonal with the imaging plane at the start of the volume but the head rapidly moved half way through the acquisition. This reveals that the slice order was interleaved with the odd slices (1, 3, 5...) acquired before the even slices $(2,4,6$...).

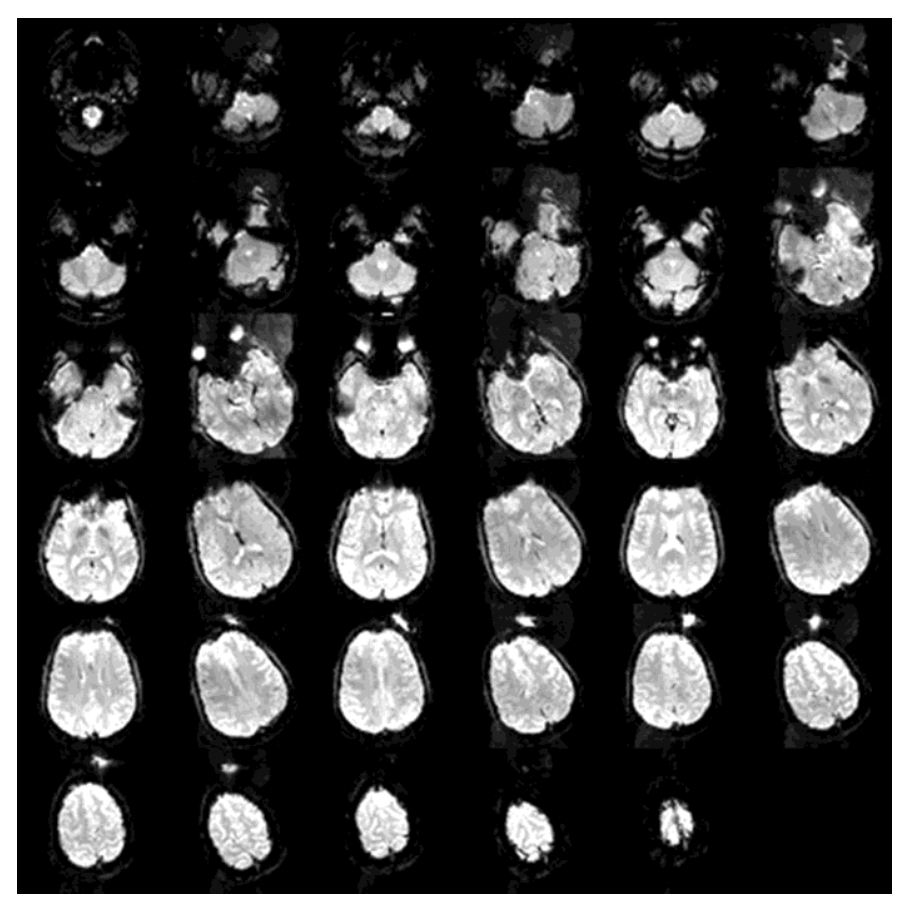

A second general issue with the Siemens format is that some important metadata are stored in the CSA header. The VR for this tag is OB (Other Byte String) and encapsulates a wide range of data encoded in Siemens proprietary format. Fortunately, the ubiquity of this format means that most modern DICOM to NIfTI converters are able to decode this information. This information is usually used to extract spatial transforms for mosaics, slice order, and diffusion gradient information. As with the multi-frame image approach, recent updates to the DICOM standard now include information, such as diffusion gradient information, as public messages. However, the inertia of vendors maintaining compatibility with the current DICOM standard, and the existence of old scanners and software versions still in use, means that there is still a strong reliance on the earlier private DICOM messages.

Siemens software computes the Image Position Patient for the mosaic images, $\left[x_{m} y_{m} z_{m}\right]$, in such a way that the parameter reflects the position with the mosaic image center shifted to the original center of the first slice. The real location for the first slice, $\left[x_{1} y_{1} z_{1}\right]$, can be the computed by Equation 3:

$$
\left[\begin{array}{l}
x_{1} \\
y_{1} \\
z_{1}
\end{array}\right]=\left[\begin{array}{lll}
r_{x} v_{r} & c_{x} v_{c} & x_{m} \\
r_{y} v_{r} & c_{y} v_{c} & y_{m} \\
r_{z} v_{r} & c_{z} v_{c} & z_{m}
\end{array}\right]\left[\begin{array}{r}
\left(m_{c}-n_{c}\right) / 2 \\
\left(m_{r}-n_{r}\right) / 2 \\
1
\end{array}\right]
$$


Where $\left[m_{c} m_{r}\right]$ are number of the columns and rows of the mosaic image, $\left[n_{c} n_{r}\right]$ are the number of columns and rows of a real slice, and others have the same meaning as those in Equation 1.

All mosaic files in a series have the same Image Position Patient, which loses the location information of other slices, so the method in Equations 1 and 2 won't work for mosaic images. To address this issue, Siemens stores a parameter, Slice Normal Vector, in the CSA header, to encode the direction cosine, $\left[s_{x} s_{y} s_{z}\right]$, at slice direction. The scaled Slice Normal Vector by Spacing Between Slices $\left(v_{s}\right)$ can replace the third column of Equation 1, to construct transformation matrix in DICOM coordinates for mosaic images, as shown in Equation 4:

$$
R_{\text {DICOM }}=\left[\begin{array}{rrrr}
r_{x} v_{r} & c_{x} v_{c} & s_{x} v_{s} & x_{1} \\
r_{y} v_{r} & c_{y} v_{c} & s_{y} v_{s} & y_{1} \\
r_{z} v_{r} & c_{z} v_{c} & s_{z} v_{s} & z_{1} \\
0 & 0 & 0 & 1
\end{array}\right]
$$

\section{Philips DICOM}

Modern Philips DICOM files often encode an entire 3D or 4D MRI series into a single DICOM file, as described by more recent extensions to the DICOM standard. In this way, DICOM images from this vendor are more similar to NIfTI images than scans from other vendors, and issues regarding correctly sorting and stacking slices are reduced. On the other hand, this structure is different from the Siemens files most familiar to the developers of the image conversion tools.

One of the unusual features with Philips data is specific to the diffusion modality. In addition to storing the raw data, these systems typically also calculate an 'istrotropic' average diffusion image and store it in the same DICOM series as the raw images. This average image can cause problems for postprocessing tools. This computed volume can be identified because it reports a non-zero b-value (tag $2001,1003)$ whereas all components of the gradient direction vector $(2005,10 B 0 ; 2005,10 B 1$; 2005,10B2) are zero. Ideally, these calculated images can be removed during the conversion to NIfTI format. Alternatively, these images can be detected and removed using the bval and bvec files generated during later analysis (as described in the Diffusion Modality section later).

Another unusual feature with multi-volume Philips data, including standard 2D DICOM, new multiframe DICOM, and PAR/REC pair data, is that the slice dimension is encoded as the last dimension. For example, the second DICOM image is the second slice of the first volume for all other manufactures we have seen, but for Philips data, it is the first slice of the second volume. This is one of the cases where Image Position Patient is needed to sort images within a series into different volumes.

\section{GE DICOM}

Relative to Siemens and Philips DICOM MR images, GE are typically the simplest. In our experience, each 2D slice is always saved as a separate DICOM file and details, such as gradient direction, are stored directly in private but generic DICOM tags (http://www.namic.org/Wiki/index.php/NAMIC_Wiki:DTI:DICOM_for_DWI_and_DTI\#Private_vendor:_GE). However, this simplicity poses some challenges as well. For example, it often results a large number of files which makes sorting process slow. Another concern with GE data is handling some of the DICOM errors in 
these files. Well documented examples include image compression errors specific to CTi devices (http://www.dclunie.com/jpegge.html). Likewise, historically some GE images report 13 bytes stored whenever 10 bytes are stored in the DICOM header (http://xmedcon.sourceforge.net/Main/Old) - since DICOM tags must have an even number of bytes, the conventional workaround is to always assume 13 means 10.

\section{Field map DICOM}

For fast echo-planar imaging (EPI) acquisitions, image distortion is often an issue. Multiple-TE field map can allow analysis tools to correct the EPI distortion to some extent. Ideally, the conversion tools should try to extract and store the required parameters for this purpose from DICOM files of different vendors, such as phase encoding direction relative to image space, Echo Time and Effective Echo Spacing. However, users should be aware that the direction of the correction also relates to the scanning sequence, as well as the way to construct the field map phase image, so it may be opposite to the phase encoding direction. The correction with wrong direction will result in worse distortion, rather than correcting the distortion. The method to check the outcome of the correction is to overlay the corrected image onto an undistorted template (e.g., the structural image). However, due to the possible head motion between EPI and structural images, one has to align the EPI image to the template. The alignment process can conceal the distortion, making it hard to visualize the effect of the distortion correction. A valid way would be to scan a phantom using the same scan protocol, and to figure out the direction of correction (Figure 3).

Figure 3: An example showing the effect of distortion correction using field map scan. The grayscale background is the structural data of an ACR phantom, and the red overlay is from a Siemens EPI scan with phase encoding direction from right to left in the picture. The left panel picture shows the original EPI, and the distortion is apparent at the lower-left corner. The middle panel shows the EPI after correction using the correct phase direction (" $y$-") in FSL which reduces the distortion. The right panel shows the result using the incorrect phase direction (" $y+$ "), which amplifies the distortion.

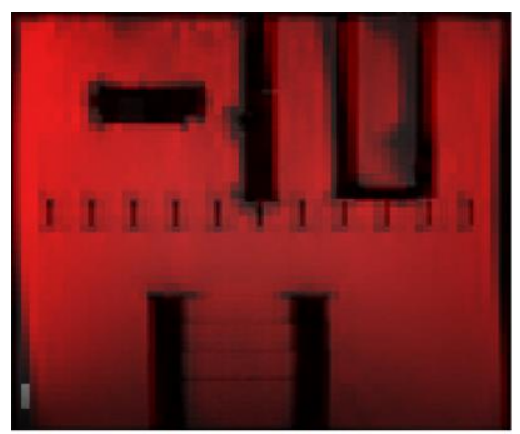

Original

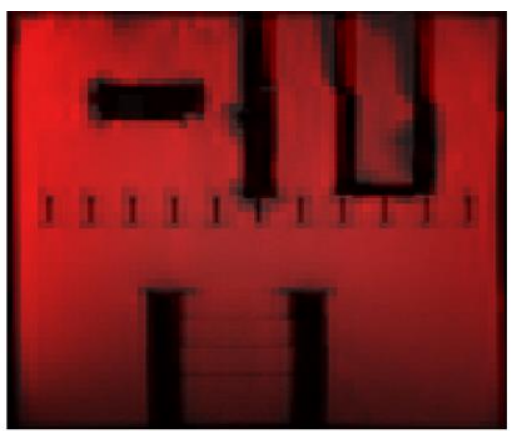

Correction with phase $y-$

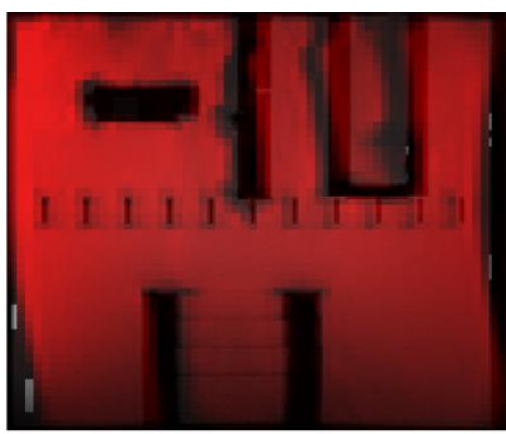

Correction with phase $y+$

\section{DICOM transfer, storage, anonymization and modification}

DICOM files are typically modified as they are transferred and archived. As described above, each medical imaging device vendor has interpreted the DICOM standard slightly differently. Likewise, 
different archiving tools create slightly different sub-sets of the DICOM standard. Traditionally, data are exported from the scanner directly to a disk (e.g. CD-ROM) or over the network to a picture archiving and communication system (PACS). Sometimes, conversion issues reflect manipulations caused by the PACS rather than the raw scan data. Therefore, if one encounters a problem converting or viewing a DICOM image, a first step is to establish whether the issue was present in the raw data from the scanner or introduced by subsequent processing.

Perhaps the most documented, prevalent and long lasting example of how a DICOM transfer can corrupt data is the files handled by many General Electric PathSpeed PACS. These systems insert a low resolution thumbnail image within the DICOM header. Unfortunately, GE implemented this thumbnail using public tags but stores the thumbnails' image data as a lossy compressed JPEG regardless of the transfer syntax of the main image. This issue was described since at least 2009, but was not patched until the Centricity PACS Version 4.0 SP10 was released in late 2014 (and will still be observed in systems that are not upgraded). Therefore, every image touched by these PACS is no longer strictly DICOM compliant. Likewise, errors in implementing the DICOM format are not reserved to one vendor - Philips initial attempts to implement the sequence (SQ) VR did not correctly implement the start and end messages. These examples demonstrate three points. First, the DICOM standard is complex, and even professional teams have difficulty ensuring complete compliance. Second, a converter that assume strict DICOM compliance may fail with some common images. Fortunately, for these two examples, the prevalence of these defects means that the popular conversion tools are likely to cope with these errors. Finally, these serve as concrete examples of how a PACS system can change the behavior of DICOM conversion.

One common desire for research is to ensure that data are properly anonymized, to ensure privacy is maintained. A DICOM file often includes a range of personal information including name, weight, date of birth, phone number and religious preference. A user may want to remove this information to ensure Health Insurance Portability and Accountability Act (HIPAA) compliance. Four popular free tools for this are the dcmtk's dcmodify (http://support.dcmtk.org/docs/dcmodify.html), DicomBrowser (http://nrg.wustl.edu/software/dicom-browser/instructions/batch-anonymizations/), PixelMed's DICOMCleaner (http://www.dclunie.com/pixelmed/software/webstart/DicomCleanerUsage.html) and the Grassroots DICOM gdcmanon (http://gdcm.sourceforge.net/html/gdcmanon.html). These tools inherently modify the DICOM header, and this can cause problems. As a concrete example, we have seen the gdcmanon and DicomVCL lose the VR of the private tags, and assign VR of Other Byte String (OB) or unknown (UN). One of the examples is Siemens private tag 0019,100A which stores the number of slices in a mosaic. This VR change can influence conversion software. While this example is easy to fix in the conversion tools, it illustrates how DICOM manipulation can disrupt image conversion.

\section{DICOM image compression}

As noted, DICOM describes dozens of different schemes for encoding image data (the transfer syntax). In contrast, the NIfTI standard always stores image data uncompressed (though some tools allow the entire NIfTI file to be compressed using the widely supported zlib file compression). Therefore, while NIfTI images may differ in image precision (e.g. storing data as 8-bit integers or 32-bit floating point) and byte order (as the format supports both little and big-endian data storage), the image 
encoding is very simple. This simplicity is a major benefit for software supporting the NIfTI format. On the other hand, software designed to aid DICOM to NIfTI conversion must deal with this wide array of possible variants.

Typically, the convention for the MRI modality appears to store the data using one of the uncompressed transfer syntaxes. Therefore, most DICOM to NIfTI conversion does not need to deal with image compression. However, many sites apply image compression when DICOM data are transferred. In our experience, the most popular DICOM compression variant is the first order lossless JPEG (1.2.840.10008.1.2.4.70), presumably because the DICOM standard defines this as the default compression method. While lossy JPEG is a very popular format for web pages and digital photography, this specific lossless JPEG format seems specific to DICOM images, generally provides poor compression ratios (Clunie, 2000), and is not supported by many of the popular JPEG libraries. Recently the DICOM standard has incorporated both lossy and lossless implementations of the JPEG 2000 standard. This format offers better compression ratios and therefore is likely to grow in popularity. Providing support for this clever but very complicated wavelet-based compression scheme is challenging because medical images are often saved with higher precision than typical in other domains (16 versus 8-bit). Currently, these high precision variations are not well supported by the application program interfaces of modern operating systems or some of the open source libraries (http://en.wikipedia.org/wiki/JPEG_2000). Given these complexities, it is unsurprising that some of the popular DICOM to NIfTI image converters do not support these compression schemes. Faced with compressed images the user must either select a converter that supports these formats (e.g. dcm2niix) or convert these files to decompressed DICOM, for example by using the DCMTK tool dcmdjpeg (http://support.dcmtk.org/docs/dcmdjpeg.html).

With regards to the personal opinion of the authors, we urge users to think carefully before deciding to archive their data using one of the compressed transfer syntaxes. While the lossy methods do tend to achieve good compression ratios with acceptable appearance to the human eye, they may lose some information that is important for statistical analyses and clinical diagnosis. On the other hand, the lossless compression schemes tend to have fairly modest compression ratios, and users may be better served by using popular and heavily supported file or disk based compression schemes.

\section{Non-DICOM formats}

The previous sections describe some of the complexities faced when converting DICOM images. However, this diversity also reflects the general success of DICOM, as it has become the dominant format for medical images. In contrast, prior to DICOM there were numerous vendor-specific formats (http://www.dclunie.com/medical-image-faq/html/part4.html). There are two cases where neuroimaging users may still encounter non-DICOM formats. First, archival studies may seek to analyze old datasets acquired prior to the DICOM standard. Second, research teams using Philips hardware may elect to export their data in the Philips research format instead of DICOM. We describe these cases below.

The dcm2nii tool can convert several legacy formats, including images in the Picker, Elscint, ECAT7, Interfile, GE Signa 4/5, and Siemens Magnetom formats. Likewise, the LONI Debabeler can support images from the DICOM, GE Signa 5, MedX, MINC, UCF, AFNI, ECAT 7, HRRT Interfile formats (Neu et al., 
2005). Since these formats actually predate the NIfTI format, users should visually inspect the resulting images to verify the spatial coordinates (e.g. make sure the data is not left-right flipped). Likewise, these conversions will not recover information about slice order.

Versions of our MRIcro software for OSX (http://www.mccauslandcenter.sc.edu/CRNL/tools/mricro) not only include dcm2niix described here, but also allow conversion of numerous other formats to the NIfTI format. For example Bio-Rad Pic, NRRD, ITK Metalmage (.mhd, .mha), AFNI (.head/.brik), Freesurfer (.mgh, .mgz) formats. In addition, this software is able to convert any bitmap image natively supported by the operating system (e.g. PNG, JPEG, etc). This is useful for confocal laser scanning microscopy as some of these systems save data as TIFF format. Again, users should visually inspect data converted from these modalities to ensure that aspects ratios, image orientation and other details have been converted properly.

Research groups using Philips scanners can export their data either as DICOM images or Philips proprietary PAR format. Each image in the latter format is stored as two files, a text header (a file with the extension '.PAR') and the uncompressed image data (a file with the extension '.REC'). As shown in Table 3, there are several tools available that support this format. Alternatively, the Matlab script r2aGUI (http://r2agui.sourceforge.net) is a dedicated tool for converting images in this format. PAR format includes all the details needed to support the full NIfTI format, including gradient directions for diffusion sequences.

\section{Diffusion modality}

Diffusion-weighted imaging is based on the random motion of water molecules. In the brain, water diffusion is anisotropic in white matter tracts: with a faster velocity along fiber paths. Therefore, by acquiring a series of images with different directional biases, one can map the brain tracts. The NIfTI standard did not specify a method for encoding these directions. However, the FSL diffusion tools (Smith et al., 2004) provided a simple text format for describing B-value amplitude (a file with the extension .bval) and gradient directions (.bvec) that has become the de-facto standard. This has now been adopted by many tools including MedInria (Toussaint et al. 2007), Freesurfer's Tracula (Yendiki et al., 2011). Other approaches include LONI MiND which stores the metadata in NIfTI extension (Patel et al., 2010).

One important feature of this format is that it requires the gradient direction relative to the image's frame of reference, whereas Philips and Siemens scanners report these directions relative to the PCS. Therefore, if one changes the slice angulation of a diffusion scan, the gradients reported in the DICOM header will not change even though the directions required by FSL do. Equation 5 shows the method to convert gradient directions from PCS $\left(\mathrm{G}_{\mathrm{PCS}}\right)$ into image frame:

$$
G_{\text {image }}=G_{P C S}\left[\begin{array}{lll}
r_{x} & c_{x} & s_{x} \\
r_{y} & c_{y} & s_{y} \\
r_{z} & c_{z} & s_{z}
\end{array}\right]
$$

The 3 by 3 matrix in Equation 5 are the direction cosines at row, column and slice directions, and the symbols have the same meaning as those in Equation 4 for Siemens mosaic image. For standard non- 
mosaic image (see Equation 1), the direction cosine at the slice direction is often computed as the cross product of row and column cosines. However, depending on the scheme of acquisition and storage, the slice stacking direction may not always follow right-handed rule, so the sign of the cross product may be opposite. The safe way is to compute this by normalizing the third column in Equation 1.

If the NIfTI image is flipped along one or more axes, the corresponding signs of $G_{\text {image }}$ need to be reversed accordingly. Note that no sign change is needed to account for DICOM and NIFTI coordinate difference, since $G_{\text {image }}$ is in image frame reference.

For files from GE, the DICOM header stores diffusion direction with respect to phase, frequency and slice encoding direction, so conversion needs to take into account whether the phase encoding direction is across rows or columns of the imaging data. Therefore, many conversion tools are able to detect diffusion-weighted DICOM images and generate FSL-format B-value and gradient directions. However, these tools tend to require information from the DICOM private tags (hence those that might change). Therefore, it is vitally important for centers to validate the gradient direction conversion for their systems (see Figure 4). We provide sample diffusion datasets and analysis scripts for images from each of the major vendors (http://www.nitrc.org/plugins/mwiki/index.php/dcm2nii:MainPage). Diffusionweighted images tend to exhibit spatial distortions, but many of these are dependent on phaseencoding direction, therefore a strategy is to acquire two sessions of DTI with opposite phase-encoding polarity allowing distortion correction (Andersson et al., 2003). For this reason, it is useful for DICOM conversion tools to record the phase-encoding direction in one of the NIfTI header text fields. 
Figure 4: Accurate tractography requires that the gradient directions are correctly specified. This image illustrates a simple test to validate this requirement. The figure shows a DTI scan acquired where the image frame of reference is rotated relative to the scanner's frame of reference (here an oblique slice plane somewhere between an axial and sagittal acquisition). The converted NIfTI image and gradient directions are processed using the FSL diffusion tools and visualized using FSLview. Here the principal diffusion vector for each voxel is illustrated as a red line. Note that these lines accurately follow the white matter tracts in all three orthogonal views, suggesting an accurate conversion of the slice vectors.

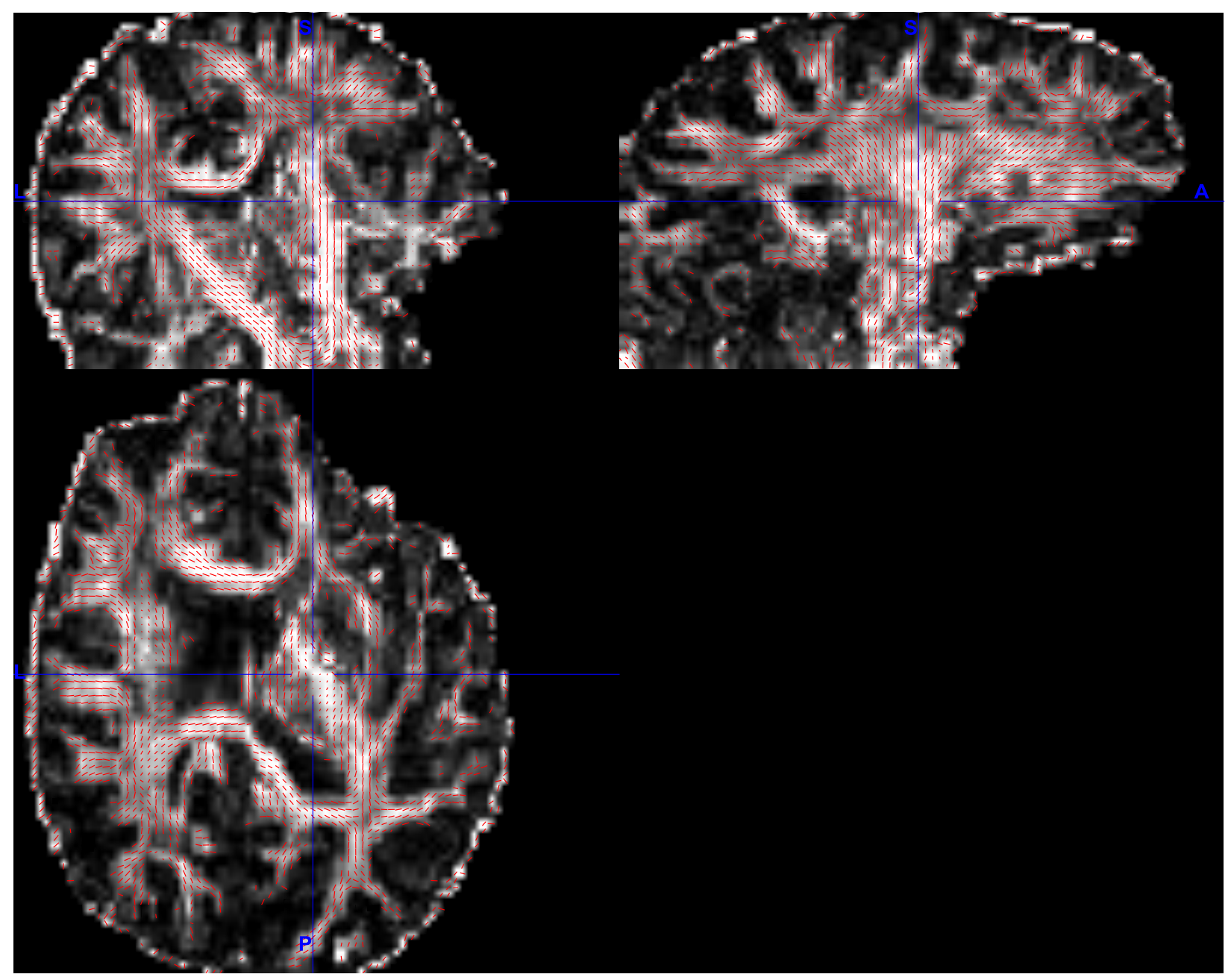

\section{Functional imaging modalities}

MRI sequences that are sensitive to blood flow (e.g. arterial spin labeling) and oxygenation levels (e.g. T2* BOLD) can be used to infer brain function. Typically, these employ EPI acquisitions to rapidly sample the 3D volume of the brain as a series of $2 \mathrm{D}$ slices. In these sequences, different locations in the brain are sampled at slightly different times, though fMRI BOLD statistics typically assume that the entire volume was sampled instantaneously. Since the hemodynamic response is sluggish, one can 
overcome this by slice time correction, which effectively infers the image intensity of a slice at one moment based on an interpolation of prior and subsequent observations. This process has been shown to improve statistical power for typical MRI sampling rates (Sladky et al., 2011). Therefore, many DICOM to NIfTI conversions attempt to detect slice order based on information in the DICOM image and record this in the NIfTI header. Unfortunately, this is not possible with all systems. For example, at the time of this writing slice timing information is not stored in the Philips DICOM header. Figure 2 illustrates a simple method for validating the slice order of EPI data. Here the participant was asked to rapidly rotate the head half way through a volume acquisition. Once this image is assembled as a 3D volume it is easy to determine the slice order based on motion. One issue with slice time correction involves multi-band sequences, where multiple spatially distant slices of a 3D volume are acquired simultaneously. For example, with a multi-band acquisition factor of 3, one would acquire three slices simultaneously, typically tripling the sampling rate. Unfortunately, the NIfTI header is not designed to handle this situation, though the multi-band factor can be recorded in the text fields of the NIfTI header. In any case, these sequences tend to have exceptionally rapid sampling rates, where the slice-time error is very small.

EPI sequences are often acquired using interleaved sequences, which reduce interference effects relative to sequential acquisitions (though they are more vulnerable to spin-history artifacts). Typically, interleaved sequences acquire all the odd slices $(1,3,5 \ldots)$ and then next acquire the even slices $(2,4,6 \ldots)$. However, users should be aware that on Siemens scanners this is not the case when the volume has an even number of slices. In this case the system acquires the even slices before the odd slices. This effect can be verified by conducting the head-rotation validation we described in Figure 2.

\section{CT Modality}

Most DICOM to NIfTI conversion tools are developed by teams with expertise in MRI. However, CT remains one of the most popular modalities in the clinic due to issues that include their speed, small number of contraindications, lower cost and suitability for resolving many clinical questions (e.g. detecting bone fractures, ability for contrast enhanced images to detect bleeds and hypoperfusion). There are now robust adaptations of the popular NIfTI MRI pipelines that allow users to normalize these images (Solomon et al., 2007; Rorden et al., 2012). Therefore, these images provide a rich opportunity for research. However, the DICOM data have three features that can confound many DICOM to NIfTI tools that must be addressed.

Many brain imaging tools assume that the image origin stored in the NIfTI header's spatial transformation matrix provides an approximate estimate of the location of the anterior commissure. Specifically, for coregistration between an individual scan and a template, the origin is used as a starting estimate. Gross errors in this position can lead to coregistration errors as algorithms become trapped in a local minimum (Jenkinson et al., 2002). For MRI, this assumption is typically fairly accurate: the origin obtained from the DICOM file refers to the scanner's isocenter, and this is likely to be close (within a few centimeters) to the anterior commissure for brain scans. In contrast, for CT images the origin listed in the DICOM file refers to the table center, which is often a meter from the anterior commissure. In this situation, it is desirable for DICOM to NIfTI converters to faithfully record this position so different series from the same individual remain in register (e.g. the enhanced and unenhanced CT scan both store the 
same coordinates), but the subsequent processing pipeline will either need manual intervention or a robust automatic method for identifying the approximate location of the anterior commissure (see Rorden et al., 2012).

The presence of gantry tilt (listed in DICOM tag 0018,1120) poses a second issue for CT brain scan conversion, particularly for step and shoot sequences (spiral sequences typically do not use a tilted gantry). CT images of the brain are often acquired with the slice axis oblique to the scanning table, so that very dense material, such as teeth fillings, won't cause image artifacts. While MRI images are often rotated with respect to the scanner bore, these volumes are always orthogonal boxes. In contrast, CT scans with gantry tilt are saved as skewed parallelogram as shown in Figure 5. In theory, this manipulation could be stored in the NIfTI header's spatial transformation matrix, but this unusual shape would not be properly handled by many tools. One concern is that some tools modify the DICOM images to correct for gantry tilt but still report the acquisition tilt in the header of the resampled DICOM image. Therefore, if the DICOM to NIfTI conversion also corrects for the tilt the resulting NIfTI image will appear skewed (in the opposite direction as the original). Hopefully, this modification is recorded in the DICOM Derivation Description tag $(0008,2111)$ and detected by the conversion software.

Figure 5: CT scans are often acquired with a gantry tilt and thinner slices near the brain stem. If uncorrected, the resulting NIfTI image can appear distorted (left). However, the image can be resampled to compensate for these effects (right).
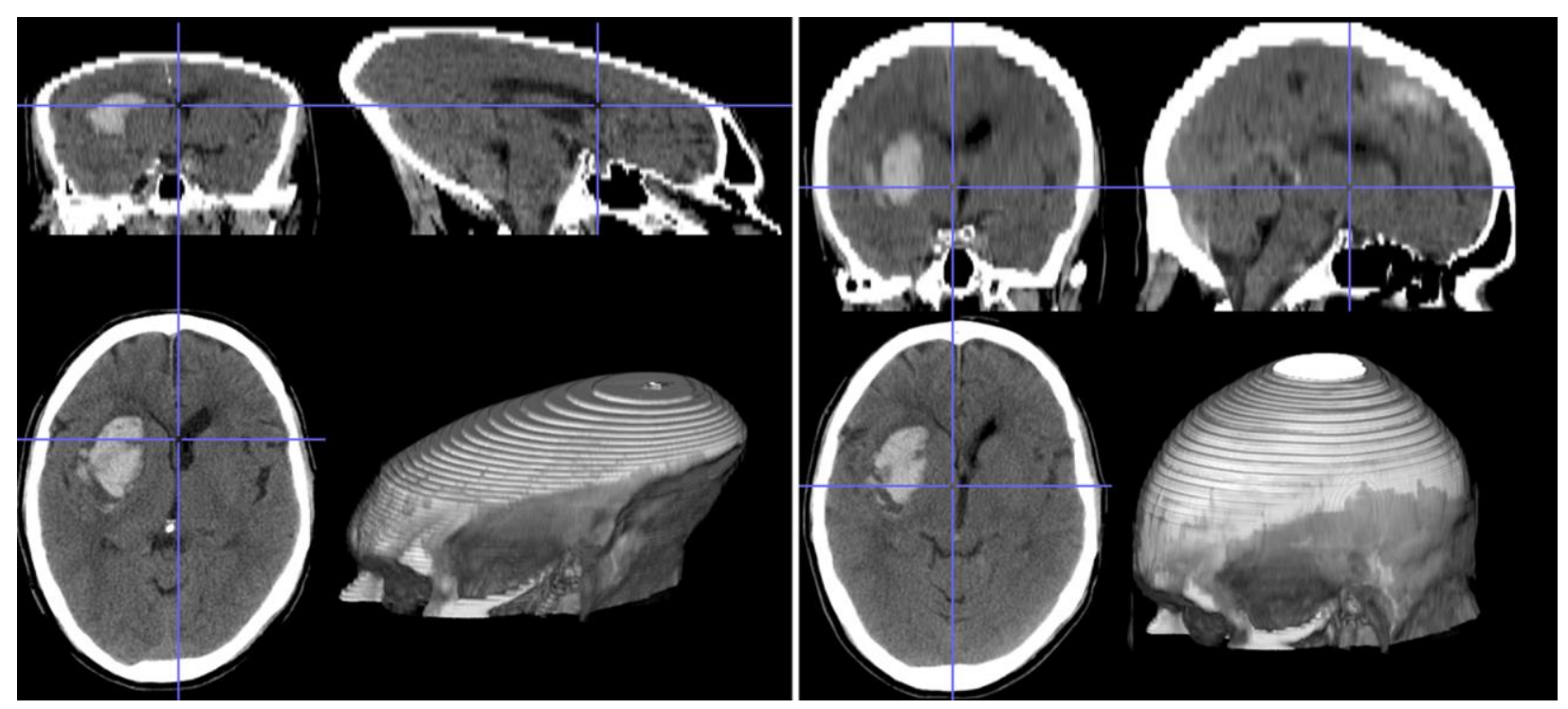

Another issue that is common for the CT modality is the fact that scans often have unequal slice thicknesses (see Figure 5), typically with thinner slices near the brain stem. This poses a challenge for the NIfTI format, which specifies that the distance between all slices is equidistant. Therefore, generating a 3D NIfTI volume from a stack of non-equidistant slices requires some interpolation.

\section{Discussion}


Ideally, users can select one of a number of tools for seamlessly converting DICOM data to the NIfTI format. In these cases, user's selection will be driven by user preference. For example, do they prefer a user interface or a command line tool? Do they prefer a stand-alone executable or do they want a MATLAB or Python-based converter that can be easily tuned for specific situations? Indeed, Table 3 focuses on tools that the authors of this work have developed and maintain, but this is not an exhaustive list. Alternatives that users may also wish to explore include dcmstack (https://dcmstack.readthedocs.org), Debabeler (Neu et al., 2005), Dimon (http://afni.nimh.nih.gov/pub/dist/doc/program_help/Dimon.html), dinifti (http://cbi.nyu.edu/software/dinifti.php), unpacksdcmdir (http://surfer.nmr.mgh.harvard.edu/fswiki/FsFastUnpackData), and XMedCon (Nolf, 2003). However, as we have described, there are likely situations where some or all of the available converters will fail to work as intended. In particular, these tools are likely to work best in common situations: using popular hardware, modalities, PACS and operating systems. When any of these variables are unusual, users should be particularly vigilant. Likewise, when one of these components is replaced or upgraded, users should consider some of the validation methods we have described. Our primary aim was to describe these situations, providing users with insight for developing a solution.

Often visual inspection is sufficient to detect when something has gone awry. In addition to visual inspection, we recommend that sites adopt automated quality assurance routines that can identify acquisition errors (e.g. slice interference, reconstruction errors), participant motion related errors as well as image conversion errors. For example, our team has developed scripts (http://www.mccauslandcenter.sc.edu/CRNL/qa) inspired by measures described by Murphy et al. (2007) and Power et al. (2012).

Ideally, the conversion from DICOM to NIfTI should record spatial orientation correctly, and this spatial transformation matrix is handled appropriately by the analysis and visualization software. However, the symmetry of the human body makes left-right errors particularly pernicious. This is compounded by the fact that some visualization tools default to neurological (left on left side) and others to radiological (left on right side) convention. A simple test is to place a fiducial marker like a Vitamin E capsule on the left template of each participant, as these are easy to identify on the highresolution anatomical scan and do not disrupt processing of other modalities.

An understanding of the conversion from DICOM to NIfTI can be helpful for identifying the source of the error. When an error occurs, a good first step is to determine whether the problem is observed in the raw data from the scanner, or only after the data has been transferred to a PACS server. The user may want to consider if their scan used unusual imaging parameters - for example having the participant lie prone rather than supine on the scanner table. The user should also examine alternative conversion tools. Examining the DICOM header from both the scanner as well as after transfer to the PACS can be useful - free tools for viewing this meta-data include Osirix (http://www.osirix-viewer.com) and DicomBrowser (http://nrg.wustl.edu/software/dicom-browser/). Finally, the developers of these conversion tools rely on their user base to supply them with these unusual images so they can identify the problems and fix the issues either in their own software or by working with the vendors involved. In particular, users with unusual images may consider contributing de-identified data to an openly 
available database that can help all developers, for example the Rosetta Bit (Yvernault et al., 2014) project (http://www.nitrc.org/projects/rosetta/).

Further, we hope the details here help future developers considering creating or extending tools for processing or converting DICOM images. Several of the tools we describe here are open source, and have been validated on a wide range of datasets and therefore provide a firm foundation for future development.

In summary, here is a list to check possible problems during DICOM to NIfTI conversion in practice, especially for the problems that may be unnoticed:

- Validation is recommended when there is a change to the scanning sequence, scanner hardware software update, and archiving system.

- Diffusion gradient sign is one of confusing parameter. Validation is recommended for a new sequence and hardware setup.

- Slice acquisition order is another confusing parameter. Depending on the setup of the acquisition scheme, the slice code reported by the converters may not be the same as the parameter from the scanner.

- If the image distortion correction requires the phase encoding direction, a validation is needed for both the field map (or similar) sequence and images to be corrected.

- If non-axial acquisition is used, special attention is needed to validate above mentioned parameters. 


\section{References}

Andersson JLR, Skare S, Ashburner J ( 2003) How to correct susceptibility distortions in spin-echo echoplanar images: application to diffusion tensor imaging. Neurolmage, 20(2):870-888, 2003.

Clunie DA (2000) Lossless compression of grayscale medical images: effectiveness of traditional and state-of-the-art approaches. Proc. SPIE 3980, Medical Imaging 2000: PACS Design and Evaluation: Engineering and Clinical Issues, 74 doi:10.1117/12.386389

Descamps B, Roggeman C, Vandemaele P, Achten E. Image Acquisition Order and Input Order of BOLDfMRI Data for Slice Time Correction in fMRI Data Processing Tools. Abstract presented at First Benelux In Vivo MR Methods Symposium.2007

Jenkinson M, Bannister P, Brady M, Smith S (2002) Improved Optimisation for the Robust and Accurate Linear Registration and Motion Correction of Brain Images. Neurolmage 17(2): 825-841.

Murphy K, Bodurka J, Bandettini PA. (2007) How long to scan? The relationship between fMRI temporal signal to noise ratio and necessary scan duration. Neuroimage. 34(2):565-74.

Neu SC, Valentino DJ, Toga AW. (2005) The LONI Debabeler: a mediator for neuroimaging software. Neuroimage. 24(4):1170-9.

Nolf E (2003) XMedCon - An open-source medical image conversion toolkit. European Journal of Nuclear Medicine. 30 (S2) S246; TP39.

Patel V, Dinov ID, Van Horn JD, Thompson PM, Toga AW. (2010) LONI MiND: metadata in NIfTI for DWI. Neuroimage. 51(2):665-76.

Power JD, Barnes KA, Snyder AZ, Schlaggar BL, Petersen SE. (2012) Spurious but systematic correlations in functional connectivity MRI networks arise from subject motion. Neuroimage. 59(3):2142-54.

Rorden C, Bonilha L, Fridriksson J, Bender B, Karnath HO (2012) Age-specific CT and MRI templates for spatial normalization. Neurolmage. 61(4):957-65.

Sladky R, Friston KJ, Tröstl J, Cunnington R, Moser E, Windischberger C (2011) Slice-timing effects and their correction in functional MRI. Neurolmage, 58(2):588-594. http://dx.doi.org/10.1016/j.neuroimage.2011.06.078

Smith SM, Jenkinson M, Woolrich MW, Beckmann CF, Behrens TE, Johansen-Berg H, Bannister PR, De Luca M, Drobnjak I, Flitney DE, Niazy RK, Saunders J, Vickers J, Zhang Y, De Stefano N, Brady JM, Matthews PM. (2004) Advances in functional and structural MR image analysis and implementation as FSL. Neurolmage, 23(S1):208-219, 2004.

Solomon J, Raymont V, Braun A, Butman JA, Grafman J. User friendly software for the analysis of brain lesions (ABLe) Comput Methods Programs Biomed. 2007;86:245-254.

Talairach J, Tournoux P. (1988) Co-planar Stereotaxic Atlas of the Human Brain: 3-Dimensional Proportional System - an Approach to Cerebral Imaging. Thieme Medical Publishers, New York, NY.

Toussaint N, Souplet JC, Fillard P. MedINRIA: Medical Image Navigation and Research Tool by INRIA. In Proc. of MICCAI '07 Workshop on Interaction in Medical Image Analysis and Visualization. Brisbane Australia, 2007.

Yendiki A, Panneck P, Srinivasan P, Stevens A, Zöllei L, Augustinack J, Wang R, Salat D, Ehrlich S, Behrens T, Jbabdi S, Gollub R and Fischl B (2011). Automated probabilistic reconstruction of white-matter pathways in health and disease using an atlas of the underlying anatomy. Front. Neuroinform. 5:23. doi: 10.3389/fninf.2011.00023

Yvernault BC, Theobald CD Jr, Smith JC, Villalta V, Zald DH, Landman BA. (2014) Validating DICOM Transcoding with an Open Multi-Format Resource. Neuroinformatics. 12(4):615-7. doi: 10.1007/s12021-014-9230-9. 